\title{
Magnetic Helicity Injection Mechanism for Double-Null Startup of the UTST Spherical Tokamak Plasmas
}

\author{
Takenori G WATANABE, Yasushi ONO, Takuma YAMADA, Shuji KAMIO, Qinghong CAO, \\ Naoto SUZUKI, Koji ISHIGUCHI ${ }^{1)}$ and Michiaki INOMOTO \\ Graduate School of Frontier Sciences, The University of Tokyo, Kashiwa 277-8561, Japan \\ ${ }^{1)}$ The Open University of Japan, Mihama 261-8586, Japan
}

(Received 29 May 2011 / Accepted 25 July 2011)

\begin{abstract}
A close relationship between magnetic helicity injection and magnetic reconnection was observed during double-null formation of spherical tokamak (ST) plasmas. The magnetic reconnection of common flux into private flux causes concentration of current density along the current sheet, forming a high eigen-value area between the helicity source (coil flux) and the ST plasma. The formation of a plasmoid and its translation to core plasma indicate intermittent translation of the high eigen-value area, suggesting a mechanism for helicity injection.
\end{abstract}

(C) 2011 The Japan Society of Plasma Science and Nuclear Fusion Research

Keywords: spherical tokamak, helicity, plasmoid, magnetic reconnection, current sheet

DOI: $10.1585 /$ pfr.6.1202131

Helicity injection is a useful method of initiating plasma current drive for various magnetized plasmas. It was developed in the spheromak project CTX[1], extended to the spherical tokamak (ST) experiment HIT [2], and is now widely used in ST, spheromak and reversedfield pinch (RFP) experiments such as the coaxial helicity source in NSTX [3] and the point-source dc helicity gun in PEGASUS [4]. Helicity injection is also observed around the connection region between poloidal coils and ST plasma during double-null merging (DNM) formation in the University of Tokyo Spherical Tokamak (UTST) plasmas. Magnetic helicity is considered to be injected from a helicity source with a high eigen-value into a helicity sink with a low eigen-value. However, helicity injection requires the helicity source, i.e. the coil flux, to be connected with the helicity sink through magnetic field lines. Thus, elucidation of how and why helicity injection is related reconnection processes is necessary. Reconnection generally produces a current sheet and plasmoids, which may directly influence helicity [5-8]. Few reports that measured the eigen value profiles of spheromaks and RFPs $[9,10]$ after their relaxation, but that of ST plasma and that during helicity injection are not yet measured. We measured for the first time 2-D eigenvalue profile of around the connecting region between the coil flux and the core plasma flux, and found that both the current sheet and the high eigenvalue plasmoids carry magnetic helicity from the coil region to the ST region.

The UTST experiment has demonstrated DNM startup of ST plasmas using two pairs of poloidal field (PF) coils outside the vacuum vessel[11-13]. In the late phase of

author'se-mail: watanabe@ts.t.u-tokyo.ac.jp
DNM formation, a single ST is connected to the external poloidal coil flux. Merging of two ST plasmas through magnetic reconnection was successfully observed by twodimensional pickup coil arrays, which are located in the $r-z$ plane of the vacuum vessel [14]. On the basis of the measured 2-D magnetic field profile, poloidal magnetic flux $\Psi$, toroidal current density $j_{\mathrm{t}}$ and toroidal and poloidal eigen values $\lambda_{\mathrm{t}}$ and $\lambda_{\mathrm{p}}$ respectively are calculated as follows: $\Psi=2 \pi \int B_{z} R d R, \lambda_{\mathrm{t}}=j_{\mathrm{t}} / B_{\mathrm{t}}, \lambda_{\mathrm{p}}=j_{\mathrm{p}} / B_{\mathrm{p}}[15]$, and,

$$
\begin{aligned}
j_{\mathrm{t}} & =\frac{1}{\mu_{0}}\left(\frac{\partial B_{R}}{\partial z}-\frac{\partial B_{z}}{\partial R}\right) \\
& =-\frac{1}{\mu_{0}}\left(\frac{1}{2 \pi R} \frac{\partial^{2} \Psi}{\partial z^{2}}-\frac{\partial B_{z}}{\partial R}\right) .
\end{aligned}
$$

Figure 1 (a) shows the experimental setup of the UTST device and the ST plasma produced in the late phase of DNM formation. Induction by the two pairs of PF coils initially ionizes the neutral gas around their double-null points and then injects toroidal plasma currents into the ST plasma. This process can increase the $\lambda$ value of the PF coil flux region than that of the ST plasma. Figures 1 (b)(d) show color contours of the poloidal magnetic flux with $j_{\mathrm{t}}, \lambda_{\mathrm{t}}$ and $\lambda_{\mathrm{p}}$ measured inside the dotted area of Fig. 1 (a) at $760 \mu$ s after PF coil discharge began. We observed for the first time in plasma experiments that a long thin high $\lambda$ region produced by the current sheet bridges the helicity source (the coil flux) and the helicity sink (the ST plasma). Both the ST plasma and the coil flux are surrounded by a common flux during helicity injection. Magnetic reconnection transforms the common flux into a private flux, forming a long thin current sheet having high $\lambda_{\mathrm{t}}$. Figures 1 (b) and (c) clearly indicate that both $\lambda_{\mathrm{t}}$ and $\lambda_{\mathrm{p}}$ in 


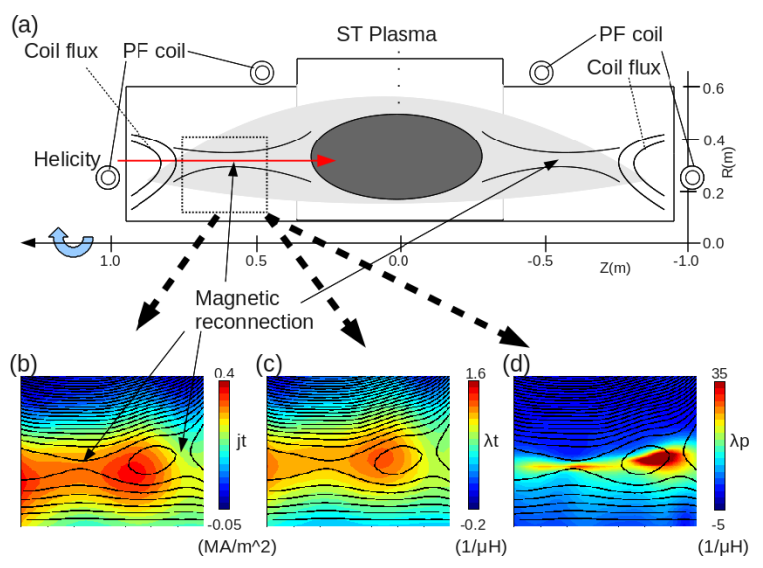

Fig. 1 (a) Experimental setup of UTST device with two pairs of PF coils on each side. Color contours of the measured 2D poloidal magnetic flux with (b) toroidal current density $j_{\mathrm{t}}$ (c) toroidal eigenvlues $\lambda_{\mathrm{t}}$ and (d) poloidal eigen-values $\lambda_{\mathrm{p}}$ at $760 \mu \mathrm{s}$.

the current sheet are approximately one order higher than those in the peripheral region. The helicity source and the helicity sink are connected by the high $\lambda$ bridge of the current sheet, enabling magnetic helicity injection from the former into the latter.

We also observed that a plasmoid having high $\lambda_{\mathrm{t}}$ that formed in the current sheet carries magnetic helicity intermittently from the coil flux to the ST plasma. Figure 2 (b) also shows the axial $\lambda_{\mathrm{t}}$ profile along the current sheet during ST startup. The plasmoid tends to have a peaked $\lambda_{\mathrm{t}}$ profile at the center of the closed flux. It has a maximum value of $\lambda_{\mathrm{t}} \sim 1.4 \mu \mathrm{H}^{-1}$ at around $z=0.53 \mathrm{~m}$ until $840 \mu \mathrm{s}$ and moves toward the ST plasma. Then, the flux decreases, but the high $\lambda_{\mathrm{t}}$ area spreads. The $\lambda_{\mathrm{t}}$ value at the entrance region of the ST plasma $(z=0.4 \mathrm{~m})$ increases from 0.6 to $1.15 \mu \mathrm{H}^{-1}$ between 680 and $880 \mu$ s because of this helicity injection and plasmoid motion. Figure 2 (c) shows the time evolutions of $\lambda_{\mathrm{t}}$ at $z=0.55 \mathrm{~m}$ and $z=0.45 \mathrm{~m}$. The plasmoid is observed to move from the former position to the latter. The $\lambda_{\mathrm{t}}$ value at $z=0.45 \mathrm{~m}$ reaches its peak of $\sim 1.3 \mu \mathrm{H}^{-1}$ about $150 \mu$ s later than that at $z=$ $0.55 \mathrm{~m}$. These facts suggest that plasmoids carry helicity from the coil flux through the bridge of the current sheet to the ST plasma. As shown in Fig. 2 (c), the plasma current increases from 90 to $125 \mathrm{kA}$ under the constant vacuum toroidal field, indicating helicity injection into the ST plasma. The peak value of $\lambda_{\mathrm{t}}$ was obtained when the largest plasmoid passed this point. The connecting region between the coil flux and the ST plasma is similar to the solar the magnetotail region [16], which tends to translate magnetic helicity from upstream in the solar wind to its downstream elongated downstream.

In summary, our 2-D eigen-value measurement indicates that helicity injection is caused by the dynamics of a high- $\lambda_{t}$ current sheet arising from magnetic reconnection (a)
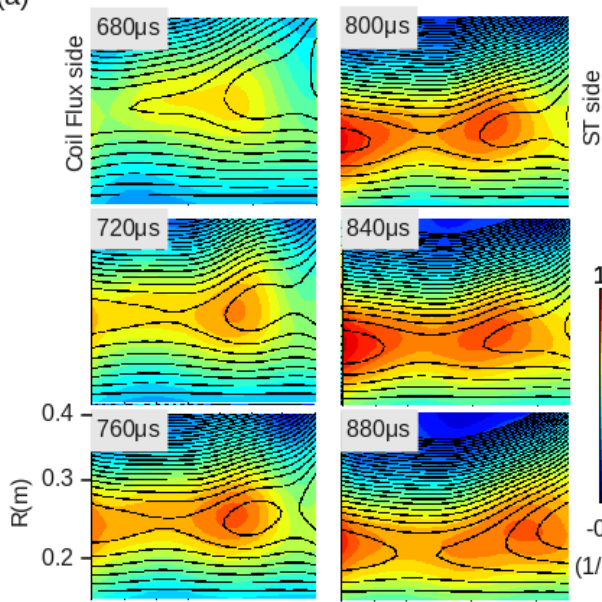

$\frac{2}{5}$

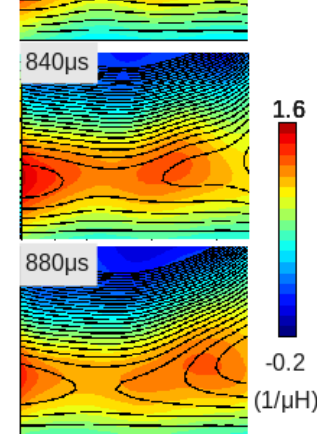

(b)
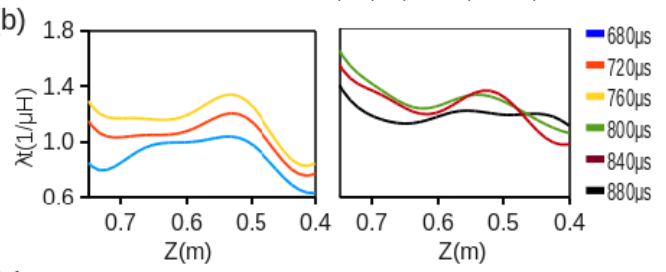

(c)

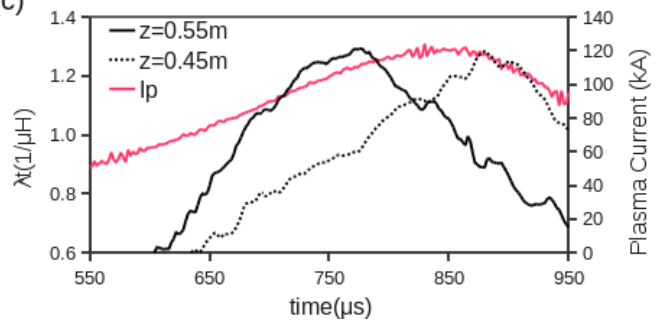

Fig. 2 (a) Poloidal magnetic flux contours with toroidal eigenvalue (color), (b) time evolution of axial $\lambda_{t}$ profile along the current sheet with the $\mathrm{x}$-point and (c) time evolution of the plasma current and $\lambda_{\mathrm{t}}$ values at $z=0.55 \mathrm{~m}$ and $z=$ $0.45 \mathrm{~m}$.

that bridges the helicity source and the ST plasma. Plasmoids with high $\lambda_{\mathrm{t}}$ are observed to grow intermittently and move toward the central ST plasma, carrying magnetic helicity. Magnetic helicity injection from the helicity source through the current sheet into the ST plasma is caused by the magnetic reconnection of common flux into private flux whose sheet current is parallel to the plasma current.

In this experiment, the relationship between magnetic helicity injection and magnetic reconnection was observed for the first time in reconnection experiments. Because we did not perform a detailed parameter scan, elucidation of how the plasma parameters change the relationship among plasmoid formation, helicity injection and Magnetohydrodynamic (MHD) and non-MHD instabilities is necessary.

This work was supported by Grants-in-Aid for Scientific Research from JSPS (22246119, 22656208, 22686085 and 21760688) and JSPS Core-to-Core Program 22001.

[1] T.R. Jarboe, Fusion Tech. 15, 7 (1989).

[2] B.A. Nelson, T.R. Jarboe et al., Phys. Rev. Lett. 72, 3666 
(1994).

[3] R. Raman et al., Phys. Rev. Lett. 97, 175002 (2006).

[4] D.J. Battaglia et al., Phys. Rev. Lett. 102, 225003 (2009).

[5] D. Biskmap, Phys. Fluids 29, 1520 (1986).

[6] J. Buchner and J.-P. Kuska, Ann. Geophysicae 17, 604 (1999).

[7] A. Bhattacharjee and X. Wang, Astrophys. J. 372, 371 (1991).

[8] K. Nishida et al., Astrophys. J. 690, 748 (2009).
[9] G.W. Hart et al., Phys. Rev. Lett. 51, 1558 (1983).

[10] S.D. Terry et al., Phys. Plasmas 11, 1079 (2004).

[11] Y. Ono et al., Phys. Plasmas 4, 1953 (1997).

[12] Y. Ono et al., Phys. Plasmas 7, 1863 (2000).

[13] A. Sykes et al., Nucl. Fusion 41, 1423 (2001).

[14] T. Yamada et al., Plasma Fusion Res. 5, S2100 (2010).

[15] Y. Ono et al., Nucl. Fusion 39, 2001 (1999).

[16] Y. Ono, K. Shibata, M. Hoshino and M. Fujimoto, J. Plasma Fusion Res. 77, 948 (2001) [in Japanese]. 